\title{
CDISC SDTM Biospecimen Events Dictionary Derived Term Terminology
}

National Cancer Institute

\section{Source}

National Cancer Institute. CDISC SDTM Biospecimen Events Dictionary Derived Term

Terminology. NCl Thesaurus. Code C124297.

Terminology associated with the biospecimen events dictionary derived term codelist of the Clinical Data Interchange Standards Consortium (CDISC) Study Data Tabulation Model (SDTM). 